\title{
A CASE STUDY ON IDEAL EXPERIMENTS OF PHYSICS
}

\author{
Chuantao Ma \\ College of Physics and Electronic Engineering \\ Taishan University, Tai'an City, China 271000
}

\begin{abstract}
Physics science is established on observation and experiment. Physics experiment method means that physicists simulate physics phenomena or natural phenomena purposefully with physics experimental equipment. Ideal experiments are the extension of physics experiment. Most of the finding of many important physics rules have important link with ideal experiment in history. Physics education must be based on experiments and take experiments as an important way for students' achievements.
\end{abstract}

Keywords- Ideal experiment, Physics experiment, Physics education

\section{INTRODUCTION}

Scientific experimental activities are commonly used for learning science in schools across the globe. Students' experience of experimental activities in elementary and secondary schools has been found to have a positive effect on the development of important abilities required for science education [1]. Physics as a natural science is established on observation and experiment. Many physical knowledge laws are obtained through scientific reasoning, induction and generalization on the basis of observation and experiment. Therefore, physics teaching must be based on experiments and take experiments as an important way for students to carry out scientific inquiry [2-4].

Physics experiment method means that physicists simulate physics phenomena or natural phenomena purposefully with physics experimental equipment. According to the design requirements, they control the secondary variables in the phenomena and show the main variables of the problem, so as to reach the objective of experiment and reveal the principles and laws in phenomena [5]. The application and population of testing method is an important milestone in the development history of physics. Practice is the only one standard to test the truth. Physics experience is an artificial physics practice, so it is a critical standard to test the correctness of the physics law. Meanwhile, physicists could have a deeper and accurate study on the physics phenomena and the physics laws behind them through experiment. Galileo was the pioneer of experimental physics since he initially presented the importance of experiment to the world. The overthrow of Aristotle's theory showed it to the world that experience should be the only one standard to test true; while physics experiment is the realest practice in physics.

Idealization method is an abstract method of scientific thought that has been vastly applied to physics study. In scientific study, scientists would artificially ignore other essence of things to highlight certain nature of the studied object. Idealization method is a scientific abstract thinking that scientists would give up the consideration on the secondary factor and mixed factors to highlight the target nature in the reality through scientific abstraction. It is the method to highlight the target nature by building up ideal model and supposing the ideal process or designing ideal experiment. The occurrence of idealization method could be tracked to the so many years ago, and it has been widely applied in the modern scientific study. For example, object would be deemed as a mass point in physics, and only the quality of the object would be considered and the shape of the object would be ignored.

Ideal experiment is also called as "hypothetical experiment". It means a special "experiment" undertaken through thinking movement. Ideal experiment is not the process but a process of deducting the process and results that experimenters make it with their own thinking based on the existing physics law under the condition of complete idealization. The "lab" of ideal experiment is human's brain, and the "test equipment" refers to people's thinking and the existing physics rules.

Ideal experiment could be the extension of physics experiment. Some experiments that could not be undertaken in the realty could be stimulated to reach similar effect through ideal experiment. However, ideal experiment is not omnipotent since the deduction of ideal experiment and the conclusion must be tested by practice. Most of the finding of many important physics rules have important link with ideal experiment in history.

The ideal experiment method is integration between idealization method and experiment method. The most distinguished feature of ideal experiment is to seize the main contradiction and special requirements of the problem and ignore the secondary contradiction to have a more profound abstract analysis based on the existing experiment; meanwhile, ideal experiment is the one undertaken under ideal condition, so it needs stricter thinking logic condition compared with the real experiment. The ignorance of the difficulty of technology and material would be better for the study on natural rule. 


\section{International Journal of Engineering Applied Sciences and Technology, 2020 \\ Vol. 5, Issue 7, ISSN No. 2455-2143, Pages 20-23 \\ Published Online November 2020 in IJEAST (http://www.ijeast.com)}

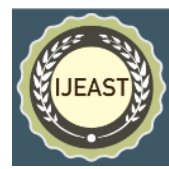

This paper focuses on the ideal experiments of physics.

\section{Classical IDEAL EXPERIMENTS IN PHYSICS}

\section{A. Galileo's Ideal Experiment}

Aristotle's theory had ruled the Europe in the $16^{\text {th }}$ century, and his theory had been deemed as truth at that time. Aristotle divided the motion (change of the object position) into two parts: natural motion and forceful motion. According to the theory of material structure proposed by him, Aristotle defined the fall of heavy objects and the rise of light objects as "voluntary motion". The object would breach the "natural motion" when the external force was applied to it, and then it would make a "forceful motion", such as rising. However, all objects would keep the status of "natural motion" when there was no external force or it had been gone. Based on it, Aristotle even explained for the motion speed of object: the object with greater weight would have greater "natural motion" trend, which results in greater speed. Seen from the aspect of modern physics, the motion theory of Aristotle could not meet the truth obviously.

It was Galileo, the father of modern science, who overthrew Aristotle's theory [6]. Galileo initially found that there should be revolution in the study method of science if he wanted to move on in physics science. Galileo linked the oldest theory and practice to create a brand-new scientific study method, ideal experiment method. The ideal experiment method enables the setup of logic deduction to be based on strict experimental conclusion, so that logic deduction would not only rely on the conclusion of experience.

Galileo designed a thought experiment. It was supposed that two heavy objects with different weights fell from the high. It could be concluded from Aristotle's theory of motion speed that a heavy object must fall faster than a small one. The two objects were linked with a rope, so the heavier object would slow down for the resistance of the lighter object; while the lighter object would accelerate for the pulling force of heavier object. Now, the two linked objects are deemed as a whole, so the speed of the whole should be between the falling speeds of the original two objects. However, seen from the aspect of whole, the whole linked by two objects should be heavier than any original two objects. Seen from the conclusion of Aristotle, the speed of the whole should be bigger than that of any original two objects, which is opposite to the conclusion just now. It could be seen that the free fall law of Aristotle could be overturned. Galileo found the internal contradiction with such ideal experiment based on logic deduction, so as to reveal the mistake in Aristotle's theory.

Being unsatisfied with the current situation and wishing to pursue the true, Galileo designed an "ideal slope" experiment. He found that it would be affected by friction in the slope experiment; and then he polished the plane to minimize the friction. The experimental tools could be extremely smooth after the special processing so as to reach the minimized friction. Based on the conclusion of the atmosphere and conclusion of previous experiment, Galileo suggested that the ball would slide down from a certain height of the inclined plane and then roll to the symmetrical slope when ignoring the friction between the ball and the inclined plane, and the maximum height that the ball could reach on the symmetrical slope is equal to the initial height of the starting slope; however, the acceleration of the ball is zero since there is no friction force when the inclined plane is horizontal, and the ball will keep a uniform rectilinear motion on the horizontal plane. The conclusion has been concluded by another great physicist to be Newton's first law of motion.

Although in reality, people could not test the conclusion of the ideal experiment with the same experiment since the air resistance and friction could not be ignored in the reality, which is the feature of ideal experiment. Galileo broke through the theory that had been deemed to be true for thousand years through several ideal experiments. Meanwhile, it also laid a foundation for the finding of Newton's first law of motion in later generation.

\section{B. Einstein's Ideal Experiment}

The theory of relativity is the most famous achievement of Einstein, but he also made great contribution in quantum theory, the kinetic theory of gases and cosmology, etc. The birth of the most famous theory of Einstein, the theory of relativity, is closely related with ideal experiment [7-9].

(1) Chasing Light Paradox

When Einstein was 16 years old, he thought of a paradox of electrodynamics that I should see such a light as an electromagnetic field oscillating and stagnating in space if I follow a light at $\mathrm{C}$ (the speed of light in a vacuum); however, it seem to be impossible according to experience or Maxwell equations. Such paradox is called as "paradox of chasing light" by researchers. The essence of the problem is the addition and subtraction of relative velocity. Photons move in a vacuum at the speed of $\mathrm{C}$ and "I" is also chasing after photons with the speed of "C", so the relative velocity between photons and "I" should be zero according to the relativity principle of classical mechanics. At this time, the light would turn to be an electromagnetic field that oscillates and stagnates in a vacuum in "my" eyes. According to Maxwell equations, the speed of light should be constant in a vacuum; however, it is also impossible for electromagnetic waves to vibrate and stagnate in the result of the hypothetical experiment. Such paradox revealed the internal contradictions in classical physics at that time. The paradox would exist forever if conversion between inertial frames would be carried out according to the classical physics transformation; however, it would mean the birth of special theory of relativity when it was used with new transformation. The paradox has contained the bud of special theory of relativity.

(2) "Train Experiment" and Special Theory of Relativity

In the end of the $19^{\text {th }}$ century, many fruitful new theories and achievements had been obtained in the field of physics, 


\section{International Journal of Engineering Applied Sciences and Technology, 2020 \\ Vol. 5, Issue 7, ISSN No. 2455-2143, Pages 20-23 \\ Published Online November 2020 in IJEAST (http://www.ijeast.com)}

but all conclusions would not get rid of Newton's absolute view of time and space. However, Einstein made a breakthrough soon and he found a relative view of time and space, so the development of physics was led to be a brandnew world.

Einstein illustrated his finding with a simple ideal experiment. It is supposed that we are on a track and two lightings are struck at the same time from the front and back of the track, the two lightings should be struck at the same time from our vision, which is a simple common sense. However, what if we do not stand on the track but a train running with a high speed when the two lightings are struck at the same time? Would they be struck at the same time? Obviously, the answer is no because the lightning in my forward direction must fall earlier than the lightning in my back direction from my vision when I have a certain speed. Hence, the lightning from my back will never catch up with me when we increase the speed of the train indefinitely, to be the speed of light, and there would be only one lightning from my vision. Two things that happen at the same time in one coordinate system will not happen at the same time in other coordinate systems, which is not compatible with Newton's absolute view of time and space. So, it would mean that Einstein set a brand-new relative view of time and space, and the special theory of relativity could build up a skyscraper with the base of the view.

\section{SIGNIFICANCE OF IDEAL EXPERIMENT}

Ideal experiment looks like a "pioneer" in the development of physics. It is always on the "frontline" of physicists to break down the barriers of their predecessors and broaden their minds, but it rarely appears in the "rear" of physicists to verify and improve theories.

\section{A. Significance of Ideal Experiment on the Development of Physics}

Ideal experiment leaves important "footstep" on the development of physics through its specialties. Ideal experiment is an important guidance of the bud of many important new theories. The experiment has shown a key role in the physics studies of both Galileo in the 17th century and Einstein in the 20th century. Ideal experiment is a critical study method to build up new concept and explore new theory in the study of physics.

(1) Ideal Experiment Is a Creative Thinking Activity

Experiment is understood as a core activity in physics education as well as physics in general $[10,11]$. Ideal physics experiment is not a practice of material, which determines that ideal experiment would be impossible to exist to test previous theory. Ideal experiment would come with suspicion, criticism and remolding of old theories. Ideal experiment could not appear from the simple conclusion of previous theories since it marks the renovation of people's minds. People's minds would be hard to surpass the previous' without ideal experiment. The "free ball experiment" of Galileo refuted the "true" existing for more than two thousand years with the method of ideal experiment; and the "paradox of chasing light" is a bud of special theory of relativity. These theories could not be generated from the accumulation of daily experience and previous theories but the direct breakthrough of the previous theories to take place of previous one.

(2)The Experimental Process of Ideal Experiment Is a Perfect Combination of Imagination and Logical Thinking

The reason why the logical thinking could be the basis of ideal experiment is to be determined by the attribute of ideal experiment. Only the abundant imagination could break through the existing thinking barrier with sufficient thinking; meanwhile, the logical thinking activity is a guaranty of the normal undergoing of ideal experiment. Only with the normal operation of logical thinking can imagination be limited on the right road. While the meticulousness of logical thinking determines the strictness of ideal experiment. Strict logic thinking activity and abundant imagination are the necessities of ideal experiment.

(3) More Important Role of Ideal Experiment in the Future

Ideal experiment is carried out with real experimental format, and it is the experiment undertaken with the strict logic thinking of physicists with the idealized and abstract method according to the existing result and conclusion of experiment. The feature of ideal experiment decides that ideal experiment would not be limited by the experimental instruments and other basic factors. Since the study content of modern sci-tech has been more and more out of the scope of experience and the theoretical system is highly abstract and divorced from intuitive experience, such ordinary thinking methods as traditional induction and deduction have been hard to be the source of innovation. Therefore, the role of creative thinking, such as scientific supposition, ideal experiment and so on, would be more and more highlighted in building up new theories. It is called a common method for scientists to understand natural science from the process to find new rules and test with experiments through experimental analysis, brave assumption, thought experiment and other methods of ideal experiment with the beginning from common sense.

\section{B. Role of Ideal Experiment in the Learning of Physics}

Ideal experiment would not only work as a weapon of "pioneer" to break through the barrier in the physics; and it could make contribution on the way of "latecomers" to move forward.

(1) Ideal Experiment Could Promote the Imagination and Thinking Ability of Students

In the teaching of physics, enhancing the teaching of experimental teaching could improve students' ability to solve the actual problems with physics problems, and they could have "physics mind" earlier. Ideal experiment is abstract, so student learning ideal experiment would be good for them to cultivate their imagination and logical thinking ability. In the teaching activity of physics, the direct physics experiment 


\section{International Journal of Engineering Applied Sciences and Technology, 2020 \\ Vol. 5, Issue 7, ISSN No. 2455-2143, Pages 20-23 \\ Published Online November 2020 in IJEAST (http://www.ijeast.com)}

could provide a more direct demonstration of experimental phenomena for students. The result of ideal experiment is not direct as the real one, but the experiment needs students to have much more creation of thinking, which would greatly simulates their creative thinking. At the same time, thinking creation of different students would gain various results. Different students would exchange their own idea and result with others under the guidance of teachers, which would greatly push them to have mutual study and effectively improve their ability of independent thinking [12].

(2) Ideal Experiment Could Enhance Students' Study Interest

Physics is a subject in need of combination of subject knowledge and physics practice. In the class, we often need to display the experimental atmosphere of partial experiment. Students' study interest would be aroused through some magic experimental atmospheres. However, in the actual class, partial physics experiment would not be shown directly for the restriction of tools. Ideal experiment needs much more thinking activity than the real experiment, so the ideal one is the best replacement in these experiments. It would be easier to arouse students' study interest than that of common experiment that teacher brings students to take part in the physics model construction of ideal experiment.

\section{CONCLUSIONS}

Ideal experiment is just a thinking process of logic deduction instead of a real experimental process. The setup of ideal experiment needs the previse physics rules or natural rules to be "material basis"; at the same time, the strict logic deduction would be "experimental method", so that the ideal experiment with the basis would be meaningful. They are necessary to the experiment. At the same time, ideal experiment could be only applied to refutation of previous theories and the proof of new logical thinking, but it could not be a judgment standard of the correctness of the theory and it could not be a standard to test the true. Oppositely, the result or conclusion of ideal experiment must be tested by practice. Physics is an experimental subject, and students must experience the process of scientific inquiry through physical experiments. Teachers should guide students to ask questions, guess hypotheses, design experiments, operate experiments, process data, analyze and demonstrate, and communicate based on the characteristics of ideal experiments. In this process, students can analyze and solve problems like physicists, deepen their grasp of physics knowledge and develop their abilities.

\section{ACKNOWLEDGEMENT}

The research is supported by the Teaching Reform Project of Taishan University (201836).

\section{REFERENCES}

[1]Kim, S. U. \& Yang, I. H. (2020). An analysis of students' cognitive bias in experimental activities following a lab manual. Education Sciences, vol. 10, No. 3, (pp. 80).

[2]Shi, W.-Z., Ma, L. \& Wang, J. (2020). Effects of inquirybased teaching on chinese university students' epistemologies about experimental physics and learning performance. Journal of Baltic Science Education, vol. 19, No. 2, (pp. 289-297).

[3]Renken, M. D. \& Nunez, N. (2010). Evidence for improved conclusion accuracy after reading about rather than conducting a belief-inconsistent simple physics experiment. Applied Cognitive Psychology, vol. 24, No. 6, (pp. 792-811).

[4]Forndran, F. \& Zacharias, C. R. (2019). Gamified experimental physics classes: A promising active learning methodology for higher education. European Journal of Physics, vol. 40, No. 4, (pp. 045702).

[5]Nigmatullin, R. R., Zhang, W. \& Striccoli, D. (2015). General theory of experiment containing reproducible data: The reduction to an ideal experiment. Communications in Nonlinear Science and Numerical Simulation, vol. 27, No. 1-3, (pp. 175-192).

[6]Hamel, J. (2009). Kepler, galileo, the telescope and its consequences. Astronomische Nachrichten, vol. 330, No. 6, (pp. 526-535).

[7]Gilmore, G. (2019). The formative years of relativity: The history and meaning of einstein's princeton lectures. Contemporary Physics, vol. 60, No. 2, (pp. 208-209).

[8]Norton, J. D. (2010). History of science and the material theory of induction: Einstein's quanta, mercury's perihelion. European Journal for Philosophy of Science, vol. 1, No. 1, (pp. 3-27).

[9]Wiseman, H. M. (2006). From einstein's theorem to bell's theorem: A history of quantum non-locality. Contemporary Physics, vol. 47, No. 2, (pp. 79-88).

[10]Ha, S. \& Kim, M. (2020). Challenges of designing and carrying out laboratory experiments about newton's second law. Science \& Education, vol. 29, No. 5, (pp. 1389-1416).

[11]Mesci, G. (2020). The influence of pck-based nos teaching on pre-service science teachers' nos views. Science \& Education, vol. 29, No. 3, (pp. 743-769).

[12]Lynning, K. H. (2006). Portraying science as humanism a historical case study of cultural boundary work from the dawn of the 'atomic age'. Science \& Education, vol. 16, No. 3-5, (pp. 479-510). 